\title{
TOPOLOGICAL GENERALIZATION OF CAUCHY'S MEAN VALUE THEOREM
}

\author{
Ivan Kupka \\ Comenius University, Faculty of Mathematics, Physics and Informatics \\ Department of Mathematical Analysis and Numerical Mathematics \\ Mlynska Dolina, Bratislava, Slovakia; ivan.kupka@seznam.cz
}

\begin{abstract}
The main goal of the paper is to introduce and study the relative derivatives for general topological spaces. We also prove a generalization of Rolle's and Cauchy's mean value theorems for real valued functions defined on topological spaces.
\end{abstract}

\section{Introduction}

In recent years notions similar to the notions of uniform convergence, monotonicity, Lipschitz condition and others have been defined in a topological way (see e.g. [5], [9], or [14]). We can use a topological approach to prove generalized mean value theorems too.

In this paper we prove a generalization of Cauchy's mean value theorem for real valued functions defined on topological spaces. To be able to use topological structures instead of the structure of the real line, we will be using the relative derivative. The classical relative derivative replaces the identity function id: $\mathbf{R} \rightarrow \mathbf{R}$ by a function $g: \mathbf{R} \rightarrow \mathbf{R}$. This notion is used in various contexts (e.g. in [2] or [10]). In this paper we define relative derivative for functions on a general topological space with values in a linear topological space. The notion "approaching infinity" will be replaced by a topological notion that could be described by the words "avoiding compacts".

We would like to thank the referee for propositions, that helped to make the article more comprehensible. The referee added also new insights by proposing Propositions 2.2, 3.2, Corollary 2.5, Lemma 2.6. and by generalizing Example 2.4.

It should be noted here that the generalized differentiation is currently developing by several mathematicians in the frame of the theory of arbitrary metric spaces. See, for example, $[1,3,6,11]$. The differentiation theory in linear topological spaces is a well-known part of analysis. Nonetheless, it seems that there were not any attempts to introduce a differentiation in topological spaces without linear or metric structures, which is the case in the present paper.

\section{Generalized derivative}

In this paper when we say "a field", we mean the fields $\mathbf{R}$ or $\mathbf{C}$. In what follows we will suppose that $X$ is a topological space, $A \subset X$ and $Y$ is a linear topological space defined over a field $F$. A function $g$ defined on $X$ is discrete on $A$ at a point $p \in X$, if there is an open neighborhood $V$ of $p$ such that the statement

$$
g(p) \notin g((V \cap A) \backslash\{p\})
$$

doi:10.5186/aasfm.2016.4120

2010 Mathematics Subject Classification: Primary 54C30, 26A24; Secondary 26A06, 26 A99.

Key words: Generalized derivatives, Cauchy's mean value theorem. 
holds. It is clear that $g$ is a discrete function (i.e. the set $g^{-1}(g(p))$ is discrete for every $p \in X$ ), if and only if $g$ is discrete on $X$ at all points. Now we define the notion of generalized derivative.

Definition 2.1. Let $p$ be limit point of $A$ and $g: X \rightarrow F$ be discrete at $p$ on $A$. A function $f: X \rightarrow Y$ has a $g$-derivative $l \in Y$ at $p$ on $A$ if for every net $\left\{x_{\gamma}\right\}_{\gamma \in \Gamma}$ of points $x_{\gamma} \in A \backslash\{p\}$ converging to $p$, the net $\left\{\frac{f\left(x_{\gamma}\right)-f(p)}{g\left(x_{\gamma}\right)-g(p)}\right\}_{\gamma \in \Gamma}$ converges to $l$.

If $l$ is a $g$-derivative of $f$ at $p$ on $A$, then we write

$$
l={ }_{g / A} f^{\prime}(p)=\lim _{x \rightarrow p, x \in A} \frac{f(x)-f(p)}{g(x)-g(p)}
$$

and ${ }_{g} f^{\prime}(p)=_{g / X} f^{\prime}(a)$ for $A=X$.

It is easy to see that, for Hausdorff spaces $Y$, a $g$-derivative ${ }_{g} f^{\prime}(p)$ if it exists, is unique.

Proposition 2.2. The following properties are equivalent for every linear topological space $Y$.

(i) $Y$ is a Hausdorff topological space.

(ii) For every topological space $X, p \in X, f: X \rightarrow Y$ and every $g: X \rightarrow F$, which is discrete at $p$, the $g$-derivative ${ }_{g} f^{\prime}(p)$ is unique if it exists.

Proof. The implication (i) $\Rightarrow$ (ii) follows from the general properties of nets in topological spaces (see, for example, [7, p. 51]). Suppose now that $Y$ is not a Hausdorff topological space. Since a linear topological space is Hausdorff if and only if this space is a $T_{1}$ space there is $y_{0} \in Y$ such that

$$
\mathrm{Cl}\left\{y_{0}\right\} \neq\left\{y_{0}\right\} \text {. }
$$

The topology of $Y$ is translation invariant, so we have also

$$
\mathrm{Cl}\{0\} \neq\{0\} .
$$

Let $f: X \rightarrow Y$ be the constant function with $f(x)=0$ for every $x \in X$. Then for every $p \in X$ and every $g: X \rightarrow F$ which is discrete at $p$ the equality

$$
{ }_{g} f^{\prime}(p)=y
$$

holds for each $y \in \mathrm{Cl}\{0\}$. The implication (ii) $\Rightarrow$ (i) follows.

Remark 2.3. It is easy to see that this new kind of derivative is a linear operator. When $X=A=Y=F=\mathbf{R}$ and $g(x) \equiv x$ we obtain the classical definition of the derivative. In general a function $f$ can have a $g$-derivative also when $f$ and $g$ are not continuous.

The following example illustrates Definition 2.1.

Example 2.4. Let $Y$ be Hausdorff and let $p$ be a point of $X$ such that $X$ is the unique open set containing $p$. If there is a $g$-derivative $l$ of a function $f: X \rightarrow Y$ at $p$ on $X$, then the equality

$$
f(x)=f(p)+(g(x)-g(p)) l
$$

holds for every $x \in X \backslash\{p\}$. Conversely, if $g: X \rightarrow F$ is discrete at $p$ on $X$ and $l$ is an arbitrary vector from $Y$ and $f: X \rightarrow Y$ satisfies (1) for every $x \in X \backslash\{p\}$, then $l$ is the $g$-derivative of $f$ at $p$ on $X$. 
Corollary 2.5. Let $Y$ be Hausdorff and let, for given $g$, a function $f: X \rightarrow Y$ have a $g$-derivative at every $p \in X$. If $X$ is a space with trivial topology, then $f$ is either injective or constant.

Proof. Let $X$ be the space with trivial topology. If there is $p \in X$ such that ${ }_{g} f^{\prime}(p)=0$ then by formula (1) $f$ is constant. Otherwise, (1) implies that $f$ is injective, because $g$ is injective as a discrete map defined on a space with trivial topology.

Lemma 2.6. Let $Y$ be Hausdorff, let $g: X \rightarrow F$ be discreet and discontinuous at $p$ on $X$ and let $f: X \rightarrow Y$ be continuous at $p$. Suppose that there exists a derivative ${ }_{g} f^{\prime}(p)$, then the equality ${ }_{g} f^{\prime}(p)=0$ holds.

Proof. Since $g$ is discontinuous at $p$, there exists $c>0$ and a net $\left\{x_{\gamma}\right\}_{\gamma \in \Gamma}$ such that $\left\{x_{\gamma}\right\}_{\gamma \in \Gamma}$ is convergent to $p$ and

$$
\left|g\left(x_{\gamma}\right)-g(p)\right| \geq c
$$

holds for every $\gamma \in \Gamma$. Since $f$ is continuous at $p$, the net $\left\{f\left(x_{\gamma}\right)-f(p)\right\}_{\gamma \in \Gamma}$ converges to $0 \in Y$. Inequality (2) implies that

$$
\left|\frac{c}{g\left(x_{\gamma}\right)-g(p)}\right| \leq 1
$$

for every $\gamma \in \Gamma$. Consequently the net $\left\{c \frac{f\left(x_{\gamma}\right)-f(p)}{g\left(x_{\gamma}\right)-g(p)}\right\}_{\gamma \in \Gamma}$ converges to $0 \in Y$. It implies that $\left\{\frac{f\left(x_{\gamma}\right)-f(p)}{g\left(x_{\gamma}\right)-g(p)}\right\}_{\gamma \in \Gamma}$ is convergent to 0 . Let $l={ }_{g} f^{\prime}(p)$. The net $\left\{\frac{f\left(x_{\gamma}\right)-f(p)}{g\left(x_{\gamma}\right)-g(p)}-l\right\}_{\gamma \in \Gamma}$ converges to 0 . Hence the constant net $\left\{y_{\gamma}\right\}_{\gamma \in \Gamma}, y_{\gamma}=l$, is also convergent to 0 . Since $Y$ is Hausdorff, it is possible only if $l=0$ holds.

The examples and assertions presented above served to illustrate some properties of the relative derivative defined on topological spaces. The reader can see, that the continuity or discontinuity of a $g$-differentiable function $f$ correlates strongly with the continuity properties of $g$. This topic will be discussed in another paper.

\section{A generalization of Cauchy's mean value theorem}

A function $g: X \rightarrow \mathbf{R}$ will be called feebly monotone at $p \in X$ on $A \subseteq X$ if for every open $O \ni p$ there exist $s, t \in O \cap A$ such that the double inequality

$$
g(s)<g(p)<g(t)
$$

holds.

The following lemma is a generalization of the fact, that if a differentiable function $f$ has an extremum at $a$, then $f^{\prime}(a)=0$.

Lemma 3.1. Let $p$ be a limit point of $A$ and let $g: X \rightarrow \mathbf{R}$ be feebly monotone and discrete at $p$ on $A$. If a function $f: X \rightarrow \mathbf{R}$ has at $p$ a $g / A$-derivative and a local extremum on $A$, then the equality

$$
g / A f^{\prime}(p)=0
$$

holds.

Proof. Suppose that $p$ is a point of local maximum of $f$ on $A$ and there is $g_{g / A} f^{\prime}(p)$. Then there exist two nets $\left\{s_{\gamma}\right\}_{\gamma \in \Gamma}$ and $\left\{t_{\gamma}\right\}_{\gamma \in \Gamma}$ of points from $A$, both converging to $p$, such that

$$
g\left(s_{\gamma}\right)<g(p)<g\left(t_{\gamma}\right) \quad \text { and } \quad \frac{f\left(s_{\gamma}\right)-f(p)}{g\left(s_{\gamma}\right)-g(p)} \geq 0 \geq \frac{f\left(t_{\gamma}\right)-f(p)}{g\left(t_{\gamma}\right)-g(p)}
$$


hold. Since the nets $\left\{\frac{f\left(s_{\gamma}\right)-f(p)}{g\left(s_{\gamma}\right)-g(p)}\right\}_{\gamma \in \Gamma}$ and $\left\{\frac{f\left(t_{\gamma}\right)-f(p)}{g\left(t_{\gamma}\right)-g(p)}\right\}_{\gamma \in \Gamma}$ converge to $g / A f^{\prime}(p)$, equality (3) follows.

Note that $g$ and $f$ from the preceding lemma are not supposed to be continuous.

The assumption " $g: X \rightarrow \mathbf{R}$ is feebly monotone at $p$ on $A$ " can not be dropped in Lemma 3.1. In fact, the following proposition holds.

Proposition 3.2. Let $p$ be a limit point of $A$ and let $g: X \rightarrow \mathbf{R}$ be discrete on $A$ at $p$. Write $\Im_{p}$ for the set of all real-valued functions on $X$, which have a local extremum and $g$-derivative at $p$ on $A$. Then the following statements are equivalent.

(i) Equality (3) holds for every $f \in \Im_{p}$.

(ii) $g$ is feebly monotone at $p$ on $A$.

Proof. The implication (ii) $\Rightarrow$ (i) is already proved in Lemma 3.1. To prove (i) $\Rightarrow$ (ii) it suffices to note that $g / A g^{\prime}(p)=1$ holds and, moreover, if (ii) does not hold, then $g \in \Im_{p}$ and (i) does not hold.

Now we give a generalization of Cauchy's mean value theorem.

Theorem 3.3. Let $(X, T)$ be an arbitrary topological space. Let $f: X \rightarrow \mathbf{R}$, $g: X \rightarrow \mathbf{R}$ be continuous functions, let $h: X \rightarrow \mathbf{R}$ be discrete and let $a, b, c, d \in \mathbf{R}$. Suppose that for every compact $K \subset X$ and every $x \in \operatorname{int}(K)$ the function $h$ is feebly monotone at $x$ on $X$ and there exist finite ${ }_{h} f^{\prime}(x)$ and ${ }_{h} g^{\prime}(x)$. If for every $\varepsilon>0$ there is a compact $K_{\varepsilon} \subset X$ such that the inequality

$$
\min \{|f(x)-a|+|g(x)-b| ;|f(x)-c|+|g(x)-d|\}<\varepsilon
$$

holds for every $x \in X \backslash K_{\varepsilon}$, then there exists $z \in X$ such that

$$
{ }_{h} f^{\prime}(z)(d-b)={ }_{h} g^{\prime}(z)(c-a)
$$

holds.

Proof. Let us define a function $p: X \rightarrow \mathbf{R}$ by the rule

$$
p(x)=f(x)(d-b)-g(x)(c-a)-(a d-b c), x \in X .
$$

Since $f$ and $g$ are continuous, $p$ is continuous too. Moreover, because of the assumptions of our theorem the following is true:

(**) For each $\varepsilon>0$ there exists a compact set $C_{\varepsilon} \subset X$ such that for all $x$ from $X \backslash C_{\varepsilon}|p(x)|<\varepsilon$ holds.

To see that $(* *)$ is true, let us consider an arbitrary $\varepsilon>0$. Denote $M:=$ $\max \{|d-b|,|c-a|, 1\}$. There exists a compact set $C_{\varepsilon} \subset X$ such that, for all $x$ from $X \backslash C_{\varepsilon}$

holds.

$$
\min \{|f(x)-a|+|g(x)-b| ;|f(x)-c|+|g(x)-d|\}<\frac{\varepsilon}{2 M}
$$

For each $x$ from $X$ the following is true:

$$
\begin{aligned}
|p(x)| & =|f(x)(d-b)-g(x)(c-a)-(a d-b c)| \\
& =|(f(x)-a)(d-b)+a d-a b-(g(x)-b)(c-a)-b c+a b-a d+b c| \\
& =|(f(x)-a)(d-b)-(g(x)-b)(c-a)| \\
& \leq|(f(x)-a)||(d-b)|+|(g(x)-b)||(c-a)| \\
& \leq(|f(x)-a|+|g(x)-b|) M
\end{aligned}
$$


and quite similarly

$$
\begin{aligned}
|p(x)| & =|f(x)(d-b)-g(x)(c-a)-(a d-b c)| \\
& =\mid(f(x)-c))(d-b)+c d-c b-(g(x)-d)(c-a)-d c+a d-a d+b c \mid \\
& =|(f(x)-c)(d-b)-(g(x)-d)(c-a)| \\
& \leq|(f(x)-c)||(d-b)|+|(g(x)-d)||(c-a)| \\
& \leq(|f(x)-c|+|g(x)-d|) M .
\end{aligned}
$$

It follows from (4) and (5) that

$$
|p(x)| \leq \min \{|f(x)-a|+|g(x)-b| ;|f(x)-c|+|g(x)-d|\} M
$$

Now we can see, that if $x$ is from $X \backslash C_{\varepsilon}$, then

$$
|p(x)| \leq \min \{|f(x)-a|+|g(x)-b| ;|f(x)-c|+|g(x)-d|\} M<\frac{\varepsilon}{2 M} M
$$

and this means that $(* *)$ is true.

Now let us distinguish two possible cases.

(i) The function $p(x)$ is constant. Then for each $x$ from $X{ }_{h} g^{\prime}(x)=0$ which means, because of the definition of $p$ that ${ }_{h} f^{\prime}(x)(d-b)={ }_{h} g^{\prime}(x)(c-a)$. Which was to be proved.

(ii) There exists $x_{0}$ from $X$ such that $p\left(x_{0}\right) \neq 0$. Suppose, that $p\left(x_{0}\right)>0$ (the case $p\left(x_{0}\right)<0$ would be treated in a similar way). Let us consider a positive number $u$ such that $p\left(x_{0}\right)>2 u>u>0$ is true.

Because of the assumptions of our theorem there exists a compact set $K_{u} \subset X$ such that for all $x$ from $X \backslash K_{u}$ we have

$$
\min \{|f(x)-a|+|g(x)-b| ;|f(x)-c|+|g(x)-d|\}<\frac{u}{M} .
$$

So for all $x$ from $X \backslash K_{u}$ we obtain $p(x)<u$ (see (6)).

This means that $x_{0} \in K_{u}$. Since $K_{u}$ is compact, the continuous function $p(x)$ has its maximum at a point $z \in K_{u}$ (with respect to $K_{u}$ ). But since $p(z) \geq p\left(x_{0}\right)>2 u$ we can see, that $p$ attains at $z$ its global maximum. Now it is quite clear that $z \in \operatorname{int}\left(K_{u}\right)$. (If $z \notin \operatorname{int}\left(K_{u}\right)$ then there exists a net $\left\{x_{\gamma}\right\}_{\gamma \in \Gamma}$ of points from $X \backslash K_{u}$ converging to $z$. We know that for all $\gamma \in \Gamma$ we have $p\left(x_{\gamma}\right)<u$. From this and from the continuity of $p$ we would obtain that the net $p\left(\left\{x_{\gamma}\right\}_{\gamma \in \Gamma}\right)$ converges to $p(z)$ and this would mean that $p(z) \leq u$, which is not possible.)

Since $z$ is an interior point of a compact subset of $X$, there exist finite derivatives ${ }_{h} f^{\prime}(z)$ and ${ }_{h} g^{\prime}(z)$. Of course, the derivative ${ }_{h} p^{\prime}(z)$ exists too and

$$
{ }_{h} p^{\prime}(z)={ }_{h} f^{\prime}(z)(d-b)-{ }_{h} g^{\prime}(z)(c-a)
$$

is true.

Since $p$ has its maximum at $z$, according to Lemma 3.1

$$
{ }_{h} p^{\prime}(z)={ }_{h} f^{\prime}(z)(d-b)-{ }_{h} g^{\prime}(z)(c-a)=0
$$

and this is equivalent to

$$
{ }_{h} f^{\prime}(z)(d-b)={ }_{h} g^{\prime}(z)(c-a) .
$$

This ends the proof.

The following Lemma, proposed by the referee, shows, how the situation simplifies under stronger conditions: if $X$ is supposed to be compact and if we are differentiating with respect to a function $h$, that is feebly monotone at every point of $X$. 
Lemma 3.4. Let $X$ be compact, let $f: X \rightarrow \mathbf{R}$ and $g: X \rightarrow \mathbf{R}$ be continuous and let at every $x \in X h: X \rightarrow \mathbf{R}$ be discrete and feebly monotone. If for every $x \in X$ there exist ${ }_{h} f^{\prime}(x)$ and ${ }_{h} g^{\prime}(x)$, then for arbitrary $a, b, c, d \in \mathbf{R}$ there is $z \in X$ such that

holds.

$$
{ }_{h} f^{\prime}(z)(d-b)={ }_{h} g^{\prime}(z)(c-a)
$$

Proof. Suppose that ${ }_{h} f^{\prime}(x)$ and ${ }_{h} g^{\prime}(x)$ exist for every $x \in X$. Note that every continuous $h: X \rightarrow \mathbf{R}$ has a global maximum at a point of $X$ and consequently such $h$ is not feebly monotone at this point. So $h$ is discontinuous at a point $z \in X$ and, by Lemma $2.6{ }_{h} f^{\prime}(z)=0={ }_{h} g^{\prime}(z)$ and we are done.

Let us remark that the proof of Theorem 3.3 contained also the proof of the following generalized Rolle's mean value theorem:

Theorem 3.5. Let $(X, T)$ be a topological space. Let $p: X \rightarrow \mathbf{R}$ be a continuous function. Let $h: X \rightarrow \mathbf{R}$ be a discrete function. Let $h$ be such, that for every compact subset $K$ of $X$ and every point $x \in \operatorname{int}(K) h$ is feebly monotone. Let for each compact subset $K$ of $X$ and for each $x$ from int $(K)$ there exist a finite derivative ${ }_{h} p^{\prime}(x)$.

If for every $\varepsilon>0$ there is a compact set $C_{\varepsilon} \subset X$ such that $|p(x)|<\varepsilon$ for very $x \in X \backslash C_{\varepsilon}$, then there exists $z \in X$ such that ${ }_{h} p^{\prime}(z)=0$.

\section{References}

[1] Ambrosio, L., and B. Kirchheim: Rectifiable sets in metric and Banach spaces. - Math. Ann. 318, 2000, 527-555.

[2] Aversa, V., and D. Preiss: Lusin's theorem for derivatives with respect to a continuous function. - Proc. Amer. Math. Soc. 127, 1999, 3229-3235.

[3] Bilet, V., O. Dovgoshey, and M. KüÇÜKaslan: Uniform boundedness of pretangent spaces, local constancy of metric valued derivatives and strong right upper porosity at a point. - J. Analysis 21, 2013, 31-55.

[4] Deimling, K.: Nonlinear functional analysis. - Springer-Verlag, Berlin, Heidelberg, 1985.

[5] Domnik, I: On strong convergence of multivalued maps. - Math. Slovaca 53:2, 2003, 199-209.

[6] Dovgoshey, O., and O. Martio: Tangent spaces to general metric spaces. - Rev. Roumaine Math. Pures. Appl. 56:2, 2011, 137-155.

[7] Engelking, R.: General topology. Revised and completed edition. - Heldermann, Berlin, 1989.

[8] Grossman, M., and R. Katz: Non-Newtonian calculus. 7th printing. - Mathco, Rockport, Massachusetts, 1979.

[9] KupkA, I.: A topological analogue of the Lipschitz condition. - Topology Proc. 32, 2008, 75-81.

[10] Light, G. L.: An introductory note on relative derivative and proportionality. - Int. J. Contemp. Math. Sci. 1:7, 2006, 327-332.

[11] Lytchak, A.: Differentiation in metric spaces. - Algebra and Analysis 16:6, 2004, $128-161$.

[12] Robertson, A. P., and W. Robertson: Topological vector spaces. - Cambridge Univ. Press, Cambridge, 1964.

[13] Taylor, A. E.: L'Hospital's rule. - Amer. Math. Monthly 59:1, 1952, 20-24.

[14] Toma, V.: Strong convergence and Dini theorems for non-uniform spaces. - Ann. Math. Blaise Pascal 4:2, 1997, 97-102.

Received 1 June 2015 • Revised received 4 September 2015 • Accepted 11 September 2015 